\title{
The Separate Neural Control of Hand Movements and Contact Forces
}

\author{
Vikram S. Chib, ${ }^{1,2,3}$ Matthew A. Krutky, ${ }^{1,2}$ Kevin M. Lynch, ${ }^{3}$ and Ferdinando A. Mussa-Ivaldi ${ }^{1,2,4}$ \\ ${ }^{1}$ Sensory Motor Performance Program, Rehabilitation Institute of Chicago, Chicago, Illinois 60611, ${ }^{2}$ Department of Biomedical Engineering and \\ ${ }^{3}$ Laboratory for Intelligent Mechanical Systems, Department of Mechanical Engineering, Northwestern University, Evanston, Illinois 60208, and \\ ${ }^{4}$ Department of Physiology, Northwestern University, Chicago, Illinois 60611
}

To manipulate an object, we must simultaneously control the contact forces exerted on the object and the movements of our hand. Two alternative views for manipulation have been proposed: one in which motions and contact forces are represented and controlled by separate neural processes, and one in which motions and forces are controlled jointly, by a single process. To evaluate these alternatives, we designed three tasks in which subjects maintained a specified contact force while their hand was moved by a robotic manipulandum. The prescribed contact force and hand motions were selected in each task to induce the subject to attain one of three goals: (1) exerting a regulated contact force, (2) tracking the motion of the manipulandum, and (3) attaining both force and motion goals concurrently. By comparing subjects' performances in these three tasks, we found that behavior was captured by the summed actions of two independent control systems: one applying the desired force, and the other guiding the hand along the predicted path of the manipulandum. Furthermore, the application of transcranial magnetic stimulation impulses to the posterior parietal cortex selectively disrupted the control of motion but did not affect the regulation of static contact force. Together, these findings are consistent with the view that manipulation of objects is performed by independent brain control of hand motions and interaction forces.

\section{Introduction}

Studies of the motor system typically focus on the production of movements. However, motor control, and object manipulation in particular, does not only concern the execution of movements but also the application of well regulated contact forces. Consider the task of writing on a blackboard with a piece of chalk. While producing handwriting motions, we must also apply an appropriate amount of contact force against the blackboard. If we press too lightly, the chalk will leave no visible trace; if we press too strongly, the chalk will break. The dual character of force and motion control is well illustrated by this example, as we control force in a direction orthogonal to the plane over which we control motion (i.e., the surface of the blackboard).

This duality is exploited in hybrid position/force control, a control scheme with a long history in the control of robot arms (Raibert and Craig, 1981; Mason, 1986; Spong et al., 1989). Hybrid control uses one system to calculate the arm joint torques to produce the desired motion in the free directions and another system to calculate the joint torques to produce the desired contact forces in the constrained directions.

Although this is a well established form of control in robotics, studies of human motor control have suggested that tasks requir-

Received Nov. 26, 2008; revised Jan. 2, 2009; accepted Feb. 3, 2009.

This work was supported by National Institutes of Health/National Institute of Neurological Disorders and Stroke Grants NS035673 and NS058300. We thank James Stinear, Jon Shemmel, and Gwyn Lewis for their discussions regarding TMS and Konrad Kording for helpful comments on this manuscript.

Correspondence should be addressed to Vikram S. Chib at his present address: Computation and Neural Systems, California Institute of Technology, Pasadena, CA 91125. E-mail: vchib@caltech.edu.

DOI:10.1523/JNEUROSCI.5856-08.2009

Copyright $\odot 2009$ Society for Neuroscience $\quad$ 0270-6474/09/293939-09\$15.00/0 ing simultaneous control of motions and forces at the hand/environment interface are achieved by a strategy that unifies the control of motions and forces. This idea is expressed by the equilibrium point hypothesis (EPH) (Feldman, 1966a,b; Bizzi et al., 1984, 1992; Latash, 1998). The EPH places within a single framework the control of posture, the generation of movements, and the production of contact forces through the control of the equilibrium position of the limb as established by the spring-like behavior of the muscles and reflexes. In this framework, the CNS may control static contact forces by shifting the same equilibrium position that guides the execution of free movements (McIntyre et al., 1995).

Although it is certain that motions and forces are coupled by the spring-like properties of muscles, it remains to be determined whether the brain controls these two entities separately or together in a single neural control module. This study considers the organization of control used by the brain during the execution of manual tasks requiring the isolated control of motions and forces and those requiring the simultaneous control of motions and forces. To this end, we tested whether the delivery of transcranial magnetic stimulation (TMS) to parietal regions of the cerebral cortex may selectively affect the cortical control of hand motions without perturbing contact force control.

Previous TMS studies have established that stimulation of posterior parietal cortex (PPC) at the onset of movement disrupts online corrections of movement plans (Desmurget et al., 1999), interferes with awareness of self-generated hand movements (MacDonald and Paus, 2003), and interferes with hand trajectories during the learning of new dynamic environments (DellaMaggiore et al., 2004). These results imply that the PPC generates 
an error signal based on the desired motion of the hand and its estimated motion. Therefore, if the control of motion and force is achieved by independent neural modules, the stimulation of PPC would result in a disruption of the neural control of motion without a direct effect on the control of contact forces. Conversely, if the control of contact forces and motions are inextricably combined, then stimulation would result in a disruption of both motion and force control tasks.

\section{Materials and Methods}

Experimental rationale

The hybrid position/force control strategy, as well as our experiments, are informed by the dynamics of the arm, which are written as follows:

$$
\tau=M(q) \ddot{q}+n(q, \dot{q})+g(q)+J^{T}(q) f,
$$

where $\tau$ is the vector of joint torques created by the muscles, $q$ is a vector of arm joint angles, $M(q)$ is the arm inertia matrix, $n(q, \dot{q})$ is a term consisting of centrifugal, Coriolis, and damping torques, $g(q)$ is a vector of gravitational torques, $J^{T}(q)$ is the transpose of the arm Jacobian matrix, relating joint angular velocities to the hand velocity vector, and $f$ is the force vector applied to the rigid environment by the hand.

Given a desired motion $q_{d}(t)$ and a desired force $f_{d}(t)$, a hybrid position force controller takes the form

$$
\tau=\tau_{\text {Motion }}\left(q, \dot{q}, q_{d}(t)\right)+\tau_{\text {Force }}\left(q, \dot{q}, f, f_{d}(t)\right) .
$$

The goal of the motion controller $\tau_{\text {Motion }}$ is to track the desired trajectory $q_{d}(t)$, and the goal of the force controller $\tau_{\text {Force }}$ is to produce the desired trajectory $f_{d}$ at the current configuration $q$.

\section{Experimental paradigm}

Eight, right-handed naive, healthy subjects (24-34 years old) participated in this study after giving informed consent in accordance with the standards of the Institutional Review Board of Northwestern University. During the experiment, subjects grasped the handle of a robotic manipulandum (HapticMASTER; FCS Control Systems) as it moved to various center out positions in the horizontal plane passing by the center of rotation of the shoulder (Fig. $1 A$ ). The arm was supported on the same plane against gravity by a low-inertia and low-friction arm support. Visual feedback of movements and applied forces were presented on a liquid crystal display monitor. Visual feedback consisted of a cursor registered to the movement of the handle of the manipulandum and an attached arrow whose magnitude and direction corresponded to the forces applied to the handle. Throughout the experiment, the subject's arm and the robot were obscured from view. To cue the application of a desired level of force, a target region for the force arrow was graphically presented. After subjects had maintained the force arrow in the target region for $3 \mathrm{~s}$, a beep was sounded to signal the request for movement initiation, and the movement servo was started. Forces applied to the handle of the manipulandum were recorded throughout the movements.

The experiment consisted of three blocks: force block, motion block, and combined block. During the force block, subjects were required to maintain $2 \mathrm{~N}$ of force in a leftward direction on the manipulandum handle as it moved along a straight path with slow constant velocity (Fig. $1 B)$. In this condition, the dominant term in Equation 1 was $J^{T}(q) f$. In this condition, inertial effects were negligible, and the quality of force control was assessed by quantifying the constancy of force vectors at different arm configurations. During the motion block, subjects were asked to track the movement of the manipulandum along a rectilinear trajectory. The handle was programmed to move with a $1 \mathrm{~s}$, bell-shaped velocity profile, which mimicked the kinematics of hand-reaching movements (Flash and Hogan, 1985). In the motion block, the dominant terms in Equation 1 are $M(q) \ddot{q}+n(q, \dot{q})$. A perfect tracking performance would result in zero interaction force between the subject hand and the handle. Thus, deviation from zero force in the motion block is attributed to errors in the subject's ability to predicatively track the handle motion by a controlled motion of the hand. The use of the interaction force as a proxy for tracking error allowed us to directly compare the subjects' performance during force and motion control tasks. Finally, the combined block was a combination of the trajectory imposed during the motion block and the force requirements of the force block. During the combined block, subjects were instructed to apply $2 \mathrm{~N}$ of force to the handle of the manipulandum as it moved to center out positions along the same paths and with the same speed as in the motion block (Fig. 1 B). The combined block was used to evaluate the simultaneous control of motion and force.

Subjects practiced each block for 60 trials, making movements along straight-line 15-cm-long trajectories to randomly ordered targets at 0, 90, 180 , and $270^{\circ}$. Experimental blocks consisted of training trials in which visual feedback was presented throughout movement and evaluation trials in which visual feedback was suppressed during movement. Trials with no visual feedback were used to evaluate performance before training, with no stimulation, to test the effect of TMS of left PPC (to disrupt the control of hand motions), and to test control stimulation of right PPC.

Experimental blocks begun with three evaluation trials in each target direction to provide a measure of baseline performance before training; next, subjects performed 15 training trials in each direction. After the training trials, subjects' behavior was evaluated for trials without stimulation, with stimulation of left PPC, and with stimulation of right PPC. During these conditions, three evaluation trials were executed in each direction. The conditions were randomized, and one training trial in each direction was presented between conditions. This procedure was 
Before

Training
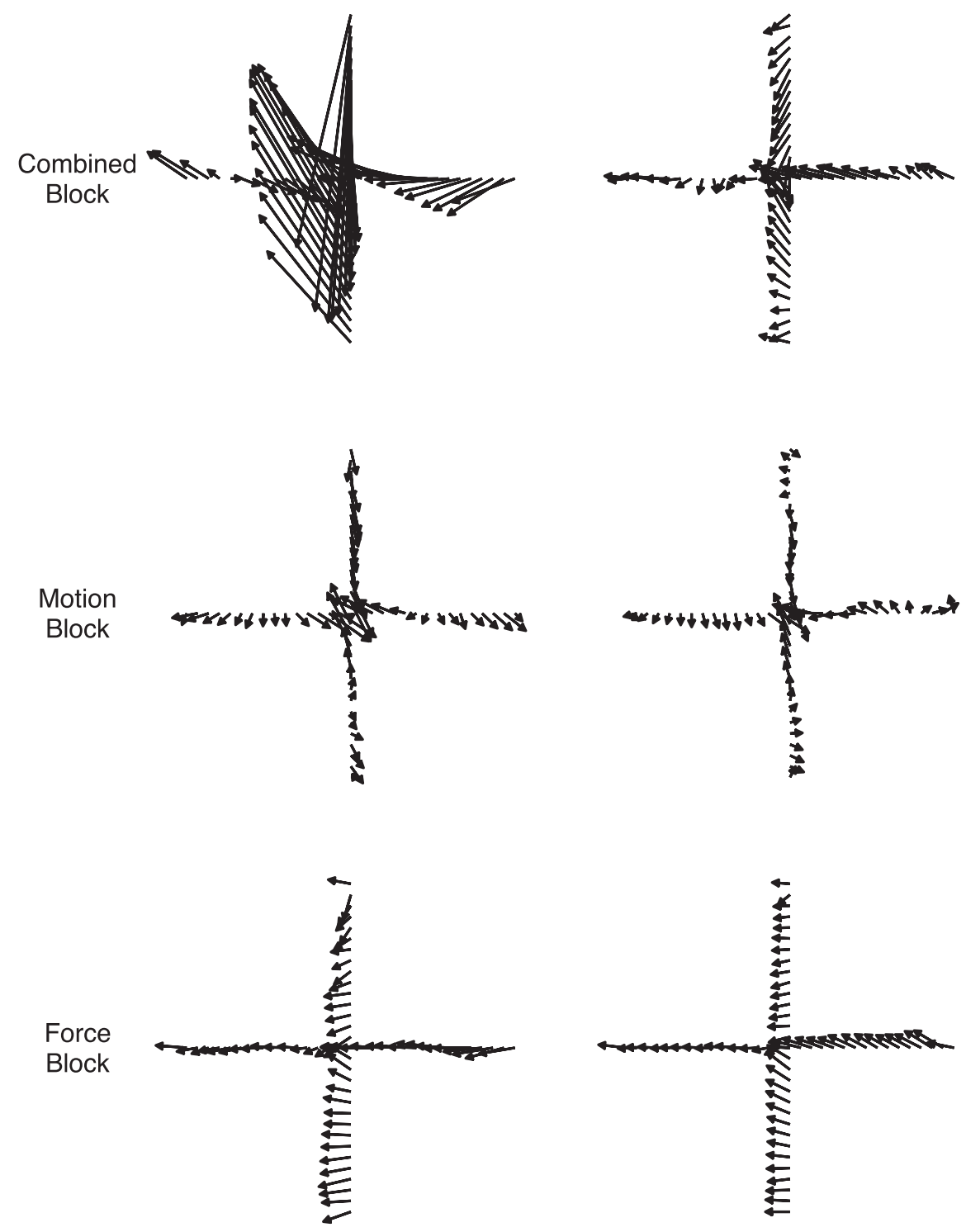

$\leftarrow 2 \mathrm{~N}$

$-2 \mathrm{~cm}$

Figure 2. Applied forces for a representative subject performing each experimental block. Force fields represent the mean of three trials in each movement direction for "no-vision" trials before training (first 12 trials) and after training (12 trials after 60 trials of practice with visual feedback).

repeated for each experimental block, and a 5 min rest was given between blocks.

\section{Control experiments}

In addition to the primary experiment, examining the differential disruption of motion control by PPC stimulation, two other control experiments were performed.

First, to observe whether TMS of PPC disrupts more complex types of force control, five subjects performed a time-varying isometric force control task. During this experiment, they were positioned as in the primary experiment, and the desired force was always in a direction away from or toward the body. Two cursors were presented to subjects: one corresponding to a time-varying desired force,

$$
\left\{\begin{array}{c}
F_{x}=0 \\
F_{r}=0.5 \times\left(1-\cos \left(2 \pi_{t_{f}}^{t}\right)\right),
\end{array}\right.
$$

where $t_{f}=2$ is the duration of the trial, and another indicating subjects' applied force. To familiarize with the task, subjects performed 25 isometric force tracking trials. After this acquaintance phase, subjects were presented with visual feedback of the desired force but not the applied force. During these trials, two conditions were presented: stimulation of left PPC and no stimulation. Each condition consisted of five trials, and condition order was randomized.

During the second control experiment, we examined muscle activations in five subjects, during each of the primary experiment blocks (i.e., force block, motion block, and combined block). Activations were recorded during three trials, in each movement direction. Muscle activity was monitored using surface electromyograms (EMGs). Reusable dual electrodes (Delsys) recorded muscle activity in the anterior, medial, and posterior deltoid, pectorals major, biceps brachii, and the long and lateral head of the triceps, and brachioradials. EMG signals were amplified (Bangoli Desktop EMG System; Delsys) with high- and low-pass cutoff frequencies of 10 and $1000 \mathrm{~Hz}$. Signals were sampled at $2 \mathrm{kHz}$ by a 32-channel, 18-bit data acquisition system (NI 6289; National Instruments). EMGs were resampled at $1 \mathrm{kHz}$ before processing.

\section{Localization of PPC and stimulation}

We obtained high-resolution anatomical images (T1-weighted, 1-mm-thick slice) of each subjects' brain using magnetic resonance imaging (MRI). We identified left and right PPC using anatomical landmarks from the MR image. The stimulation sites included the intraparietal sulcus and the adjacent cortex in the superior and inferior parietal lobule. Stereotaxic coordinates (Talairach and Tournoux, 1998) for left PPC $(x=-36, y=-64, z=54)$, obtained from previous studies (Chouinard et al., 2003; MacDonald and Paus, 2003), were used to validate anatomical localization of the stimulation site. The putative homolog of this site in macaque monkeys is the medial bank of the intraparietal sulcus (Rushworth et al., 2001; Andersen and Buneo, 2002).

After labeling stimulation sites on the MRI, we used frameless stereotaxy to localize the stimulation sites on the scalp. Localization of scalp positions overlaying the stimulation sites was facilitated by a three-dimensional infrared optical system and Brainsight software. Using this system, we converted the anatomical coordinates from the MR image into each subject's native coordinate space. Frameless stereotaxy allowed us to track the position of the coil throughout the experiment.

Single-pulse TMS was applied at an intensity of $120 \%$ of resting threshold. Resting threshold was defined as the intensity of stimulation necessary to induce a $50 \mu \mathrm{V}$ motor response (as recorded from surface 
EMG) in the first dorsal interosseus (FDI) of the right-hand, in response to 5-10 consecutive stimuli (Rossini et al., 1994). For this purpose, the center of the coil was located at the optimal location for producing motor responses in right FDI. The coil was held tangential to the scalp above motor cortex. To reduce the possibility of activating motor cortex, parietal stimulation was applied with the stimulator aligned at an oblique angle, with the central windings parallel to the central sulcus. During the experiment, pulses were applied $40 \mathrm{~ms}$ after the onset of movement. Timing was based on previous studies demonstrating that TMS applied to parietal areas had sizable effects on online error detection (Desmurget et al., 1999) and learning of dynamics (Della-Maggiore et al., 2004). All stimuli were delivered using a Magstim 200 magnetic stimulator and a figure-ofeight coil.

At the end of the experiment, for five subjects, EMG was recorded from the FDI while the entire limb was in moderate tonic contraction, and PPC was stimulated at a frequency of 0.5 Hz. EMG analysis involving the FDI confirmed the absence of TMS-related muscle activation during PPC stimulation. This finding is consistent with other work showing that PPC stimulation does not induce muscle activations (Desmurget et al., 1999; Tunik et al., 2005).

To control for collateral auditory and tactile effects of stimulation, during training trials, evaluation trials with no stimulation, and stimulation trials, a sham coil was placed on edge orthogonal to the figure-of-eight coil. The orthogonal coil was discharged at movement onset to ensure consistent auditory experimental conditions.

\section{Data analysis}

Learning measure. To evaluate performance, an average norm was calculated between the applied level of force $F_{\text {applied }}$ and the target level of force $\hat{f}$.

$$
\frac{\int_{t_{f}}^{t_{i}}\left\|F_{\text {applied }}(x, \dot{x}, t)-\hat{f}\right\| d t}{t_{f}-t_{i}}
$$

This measure describes error between subjects' applied force and the target level of force application.

Field interpolation. As subjects were servoed through trajectories, forces applied to the handle of the manipulandum were measured. These measured force vectors were considered to be a sample of a continuous force field describing the operation of the neural controller. In this experiment, a force field is simply characterized as a function relating every workspace location to a corresponding force vector (Giszter et al., 1993). We used piecewise linear interpolation to estimate the force fields beyond the sampled locations and velocities. This procedure involved partitioning the servoed trajectories into equally spaced nonoverlapping segments, $1 \mathrm{~cm}$ in length. Applied forces were linearly interpolated at the endpoints of these segments.

We generated interpolated force fields $F_{\text {Combined }}, F_{\text {Motion }}$, and $F_{\text {Force }}$ for trials before training, after training, during PPC stimulation, and control stimulation, for each experimental block. Trials from each of these phases were averaged to provide a mean measure of the force field.

Field combination. Using the estimated fields, we were able to test the hypothesis that the nervous system uses hybrid control during the simultaneous control of motions and forces. Consistent with this hypothesis are the following predictions. (1) Performance in the combined block can be decomposed into the independent behaviors of the motion and force blocks:

$$
F_{\text {Combined }}(x(t)) \cong c_{\text {Motion }} F_{\text {Motion }}(x(t))+c_{\text {Force }} F_{\text {Force }}(x(t)) .
$$

(2) TMS disruption of $F_{\text {Combined }}(x(t))$ could be attributed to the selective TMS disruption of $F_{\text {Motion }}(x(t))$. This vector summation hypothesis (Eq. 4) provides the most fundamental rule of combination, by which controllers may act independently and not interfere with one another (supplemental information, available at www.jneurosci.org as supplemental material). It is important to realize that, because the forces recorded from the experiment were in Cartesian space, we chose to express them as spatially interpolated fields of force. However, the combination hypothesis (Eq. 4) is compatible with the combination of controllers shown in Equation 2. The torque controllers of Equation 2 are simply related to the 
A

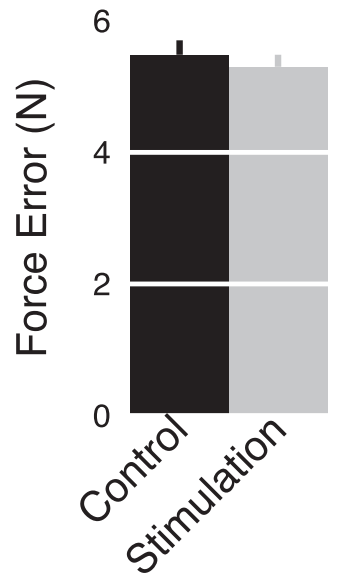

B

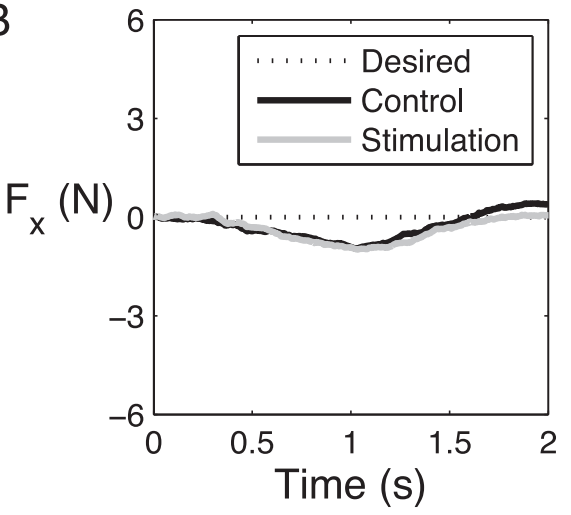

C

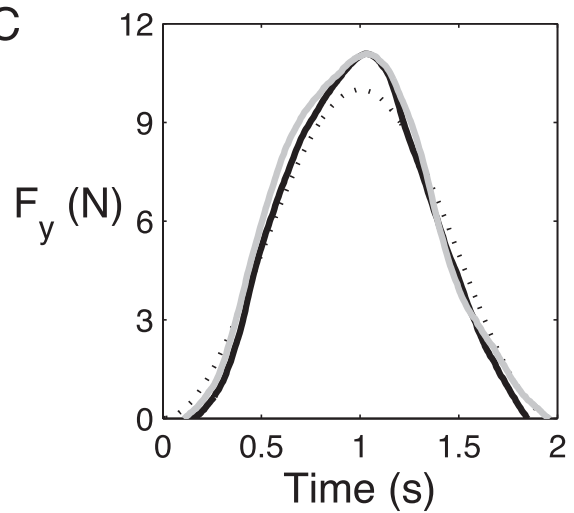

Figure 4. TMS of PPC does not disrupt more complex force control. $\boldsymbol{A}$, Mean force error for no-vision trials without stimulation and with PPC stimulation. Error bars denote SEM. $\boldsymbol{B}, \boldsymbol{C}$, Force profiles for a representative subject performing the isometric force tracking task.

measured Cartesian forces at the hand by the Jacobian of the forward kinematics, $J^{T}$.

To assess how well the combination describes the behavior, we used the following error measure:

$$
R^{2}=\frac{\left\|F_{\text {Model }}\left(x_{i}\right)-\bar{F}_{\text {Combined }}\right\|}{\left\|F_{\text {Model }}\left(x_{i}\right)-\bar{F}_{\text {Combined }}\right\|+\left\|F_{\text {Combined }}\left(x_{i}\right)-F_{\text {Model }}\left(x_{i}\right)\right\|} .
$$

This measure reports a value between 0 and 1 that represents the percentage of variation in magnitude and direction of the forces described by the model, $F_{\text {Model }}$, that can be explained by subjects applied force, $F_{\text {Combined }}$.

EMG analysis. EMG data for each muscle were processed by removing the mean, normalizing to the EMG recorded during a maximum voluntary contraction, rectifying, and finally averaging across a block. In turn, mean muscle activation was calculated for each experimental condition by averaging mean activations across muscles.

\section{Results}

\section{Unperturbed behavior}

Figure 2 shows a typical subject's applied forces represented as force fields (for a description of force field generation, see Materials and Methods). These fields illustrate trials before training and after training, during the combined, motion, and force experimental blocks. To facilitate the emergence of predictive tracking, subjects were presented with visual feedback of hand position and applied force while executing repeated trials in each movement direction. During the combined block, before training, large discrepancies were found between the desired force and subjects' applied force. Although errors were reduced after training, there was still a marked difference between desired and applied forces. During the motion block, before training, subjects' applied forces were markedly different than the desired zero force. After training, force errors were reduced, and subjects' applied force vectors were closer to the target zero force. However, there were still significant errors between the desired level of force and subjects' applied forces. The force block showed small errors between the desired and applied forces before training, and these errors were almost completely reduced after training.

Group statistical analysis of force errors in the three tasks (Fig. $3 A$ ) shows that, after practice, subjects reduced the force error in the combined $(t=2.740 ; p<0.05)$ and motion $(t=3.940 ; p<$ 0.01 ) blocks. The effects of practicing the tasks are demonstrated by the drop in mean force error after training (blue bars) compared with the initial performance (green bars). This suggests that subjects learned through practice to produce the required level of contact force. In the case of the motion task, the ability to maintain a minimal level of contact force after training implies that subjects learned to track the predicted motion of the handle more accurately. Although the learning effect was most significant in the motion task, the error in the force task also decreased slightly with training, but the change was not significant $(t=$ 1.752; $p=0.062$ ). This suggests that fluctuations associated with the control of motion are the primary source of force variability in the motion and combined tasks.

\section{TMS disrupts the neural control of motion but not force}

Stimulation of PPC (red bars) resulted in significantly higher mean errors in the combined block $(t=-5.220 ; p<0.001)$ and the motion block $(t=-6.029 ; p<0.001)$ but not in the force block $(t=0.730 ; p=0.2444)$ (Fig. $3 A$ ). Force error profiles from the combined and motion blocks indicate that TMS stimulation of left PPC resulted in a systematic increase in error in large portions of the movement (Fig. 3B). However, increases in error were not observed in the profiles of the force block. TMS stimulation did not result in higher levels of mean force error during the force block. This is consistent with the hypothesis that interaction forces are mediated by different cortical control mechanisms than those associated with the production of hand motions.

To further test the effect of TMS of PPC on the control of contact force, we stimulated subjects while they performed a more complex task, in which they were required to track a timevarying force. Figure $4 A$ illustrates that TMS of PPC during this more complex version of force control does not disrupt behavior $(t=1.261 ; p=0.276)$. The force tracking errors in stimulation and control trials are essentially equivalent and the temporal profiles of the force components (Fig. $4 B, C$ ) produced by a typical subject performing this task also illustrate that behavior is not affected by the TMS.

These results suggest that the stimulation of PPC disrupts the control of hand motions but not the control of static hand contact force. This observation is consistent with other TMS studies of reaching movements, in which the stimulation of PPC disrupted the development of dynamic motor errors used to correct an ongoing trajectory (Desmurget et al., 1999). In contrast, our findings are inconsistent with theories of motor control (McIntyre et al., 1998) that link the control of contact force to the control of hand motion. 
A combination of motion and force fields describes combined control

We next expressed behavior in each experimental block as fields of force. The fields $F_{\text {Motion }}, F_{\text {Force }}$, and $F_{\text {Combined }}$ allow us to examine the motor output of the motion controller, force controller, and the combined control in a common framework. In this framework, measured force vectors are treated as samples of a continuous force field describing the operation of the neural control system. If the latter were composed of multiple modules operating concurrently, as is suggested by our aforementioned results, the forces generated by each module at each time and at any given spatial location would contribute additively to the net force acting on the limb. This is a simple composition mechanism supported by basic principles of mechanics (see Materials and Methods, Eq. 4).

Figure 5 shows force fields generated by a subject performing each experimental block. It is apparent that TMS to PPC resulted in higher forces and a disruption of behavior in the combined and motion blocks but not in the force block. The bottom right panel shows the prediction of a model (Eq. 4) in which the forces recorded during the motion and force blocks are linearly combined point by point. The forces obtained in each block are scaled by two coefficients, whose two numerical values were selected so as to minimize the difference between the resulting linear combination and the data in the combined field. Both immediately after training and after TMS, this linear combination of the motion and force fields describes the combined field, with summation coefficients that are close to unity: $c_{\text {Motion }}=0.86, c_{\text {Force }}$ $=1.2$. Unit values of the coefficient are particularly significant because they correspond to a simple law of vector summation, which in turn would reflect a high degree of functional independence of the underlying control systems.

The ability to independently control motions and forces appeared to evolve with practice. The summation coefficients were broadly distributed $(0.974 \pm 0.80)$ before training (Fig. $6 A)$, and the $R^{2}$ for these combinations was $\sim 0.5$ (Fig. $6 B$ ). Thus, a simple summation of behaviors does not adequately describe the combined behavior before training. After training, however, PPC stimulation, and control stimulation, coefficient values were $0.981 \pm 0.15$. No significant difference was found between these values and $1.0(t=-0.838 ; p=0.406) . R^{2}$ values for coefficients of combination after training, for PPC stimulation, and control stimulation were $\sim 0.88$, indicating that the summation of motion and force controllers adequately describes the combined task (Fig. 6B). These findings are consistent with a hybrid control strategy that, after practice, combines modules of control without scaling. Furthermore, after training, the fields $F_{\text {Motion }}$ and $F_{\text {Force, }}$ share equal amounts of variance in the field summation, suggesting that each controller contributes equally in describing the 0.90).
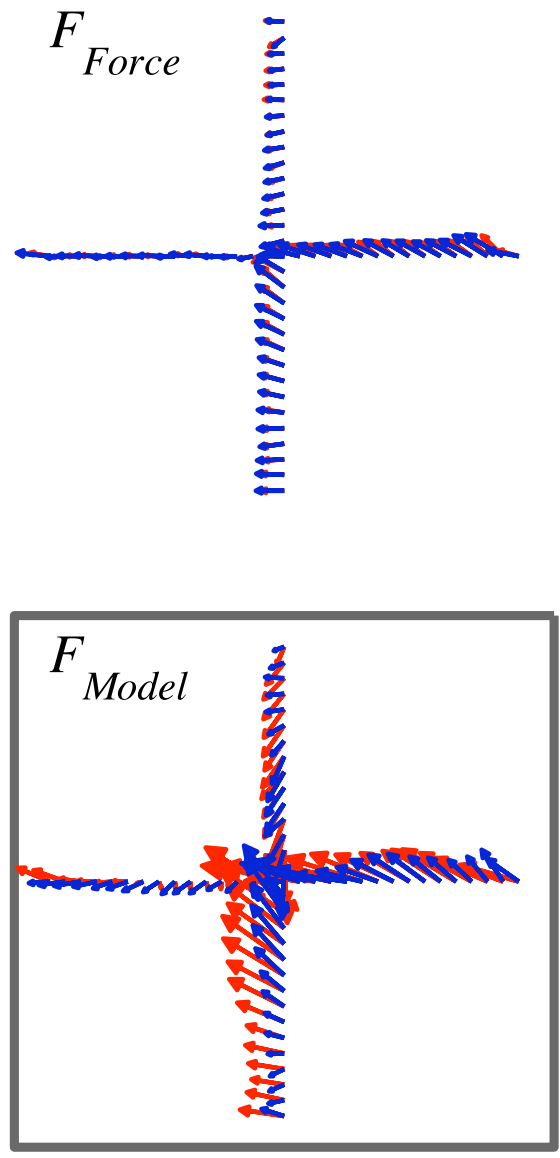

\section{$\leftarrow 2 \mathrm{~N} \quad \leftarrow$ After Training $-2 \mathrm{~cm} \leftarrow$ Stimulation}

Figure 5. A linear summation of independent motion and force controllers describes the combined task. Fields of force shown (t) motion (top left), force (top right), and combined (bottom eft) experimental blocks. Vector summation of the $F_{\text {Motion }}$ and $F_{\text {Force }}$ fields for after training and during PPC stimulation Fields (bottom right) (after training, $c_{\text {Motion }}=0.89, c_{\text {Force }}=1.04, R^{2}=0.88 ; \operatorname{PPC}$ stimulation, $c_{\text {Motion }}=0.86, c_{\text {Force }}=1.20, R^{2}=$

combined task (Fig. 6C). It is remarkable that this summation mechanism was not present in the naive subjects but was expressed as they acquired proficiency in the task.

The significantly higher force errors produced by stimulation during the combined block are described by the combination of motion and force fields during stimulation. Given that (1) values of the regression coefficients point to a summation of controllers and that (2) the force block is unaffected by TMS stimulation, increased errors exhibited during stimulation of the combined block are fully accounted for by the errors resulting from TMS during the motion block. As in trials after training, variance is equally accounted for by each controller in the stimulation case.

\section{Mean EMG activations across blocks}

The effects of TMS of motor cortex have been found to be activity dependent: the greater the level of voluntary contraction, the greater the motor-evoked potential induced by stimulation (Semmler and Nordstrom, 1998). Although we did not stimulate motor cortex in this study and found no muscle activity induced by stimulation of PPC, we wanted to ensure that muscles were in 

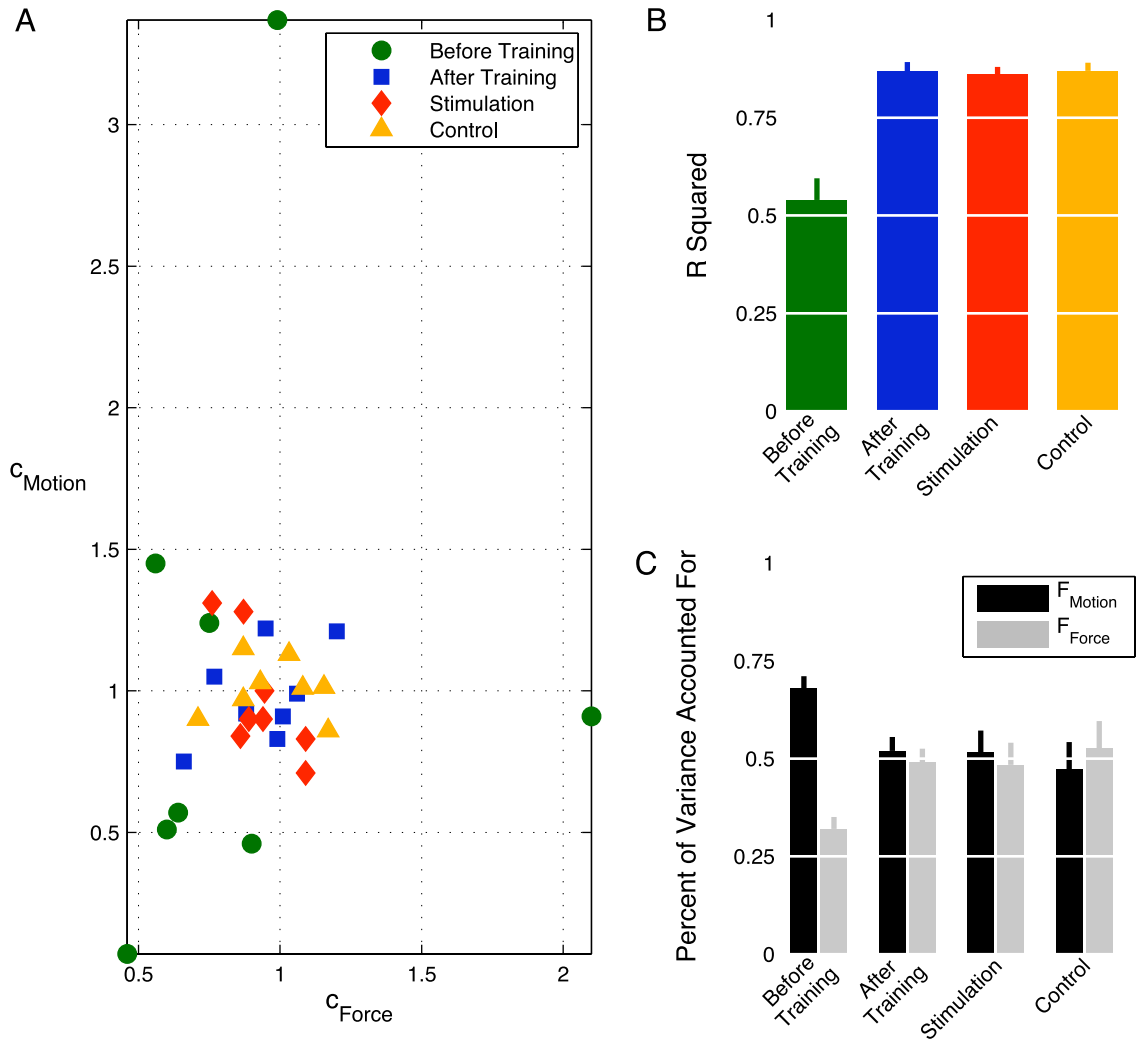

Figure 6. Motion and force controllers equally contribute to the combined behavior. $\boldsymbol{A}$, Summation coefficients for the field combination hypothesis. Field combination is performed on fields before training, after training, during PPC stimulation, and control stimulation. $\boldsymbol{B}$, Variance accounted for by each controller for each vector summation. $\boldsymbol{C}, R^{2}$ for each field combination. Error bars denote SEM.

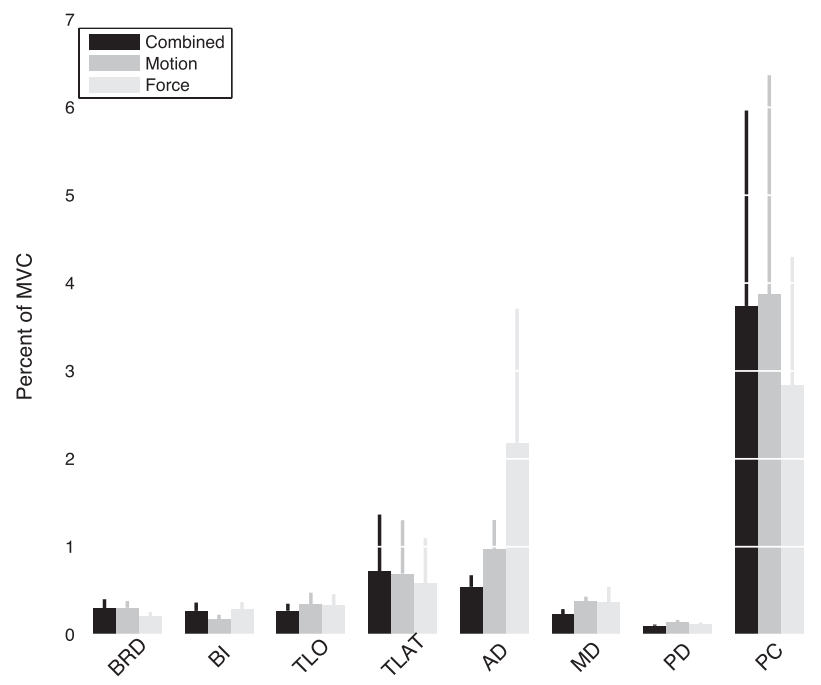

Figure 7. Muscle activation is similar for all experiment blocks. Bars denote mean muscle activation across subjects for each experiment block. Error bars denote SEM. BRD, Brachioradials; BI, biceps brachii; TLO, long head of the triceps; TLAT, lateral head of the triceps; $A D$, anterior deltoid; MD, medial deltoid; PD, posterior deltoid; PC, pectorals major.

a similar state of activation during each experimental condition. This would reduce the possibility that the variable effects of TMS were a result of differing muscle activations during each condition.

Figure 7 shows no significant differences in muscle activations between experimental conditions. Although there was increased activation in anterior deltoid and decreased activation in pectoralis major during the force block compared with the combined and motion blocks, these differences were not significant (Table 1 ). These results illustrate that, during the experimental blocks (i.e., motion, force, combined), the states of muscle activity were similar. Accordingly, the smaller effect of TMS during force blocks, relative to motion blocks, could not be attributed to a larger level of baseline muscle activity during movement compared with the generation of quasistatic forces.

\section{Discussion}

Together, our results lead us to conclude the following: (1) the posterior parietal cortex is critical for the neural control of hand motions but not for the regulation of interaction forces; (2) the nervous system uses a hybrid motion/force control strategy in tasks that involve mechanical contacts with the environment; and (3) practice of tasks involving contact forces and motions enhances the mutual independence of force and motion control. The latter conclusion is supported by the observation that, after training in tasks involving the concurrent control of motions and forces, subjects learned to generate the desired performance by a direct summation of motion and force control policies.

To identify these control policies, subjects were required to track the movement of a robotic manipulandum. The experimental design necessitated subjects' arm movements to be constrained to those of the manipulandum and antigravity orthosis. Nevertheless, the conclusions from this study may be generalized to the neural control of arm movements in a broad variety of contexts, including, but not limited to, movements in free space.

In our experiments, to characterize neural control, we recorded subjects' fields of force in Cartesian space. The Jacobian of the forward kinematics relates these measured force fields to the joint torques created by the muscles. Equation 1 describes both free and constrained arm movements and the resulting joint torques produced by the muscles. In this formulation, the force field describing control of arm motion can be equated to the joint torques produced by the terms $M(q), n(q, \dot{q})$, and $g(q)$; the field describing force control at the hand/environment interface is embodied in the last term, $J^{T}(q)$. In this way, force fields can be equated to the necessary joint torques to produce voluntary reaching movements.

In free space, the environment does not apply any force to the moving arm, and the brain is free to generate any desired motion consistent with body mechanics. The opposite, or "dual," condition is encountered when pushing against a wall. In this situation, the environment (the wall) constrains the position of the hand, and the brain has complete authority on the contact force. Free space and rigid constraints are two extremes in a spectrum of arm/environment interactions. The presence of separate modules for acting in these two extremes is likely to be the simplest way to facilitate learning movements within intermediate environments. Control in these variable impedance environments can be achieved by the appropriate combination of force fields generated by each controller. 
Table 1. EMG statistics

\begin{tabular}{ll}
\hline Muscle & ANOVA across blocks \\
\hline Brachioradialus & $F_{(2,12)}=0.515 ; p=0.610$ \\
Biceps brachii & $F_{(2,12)}=0.540 ; p=0.597$ \\
Long head of the triceps & $F_{(2,12)}=0.115 ; p=0.892$ \\
Lateral head of the triceps & $F_{(2,12)}=0.014 ; p=0.986$ \\
Anterior deltoid & $F_{(2,12)}=0.880 ; p=0.440$ \\
Medial deltoid & $F_{(2,12)}=0.532 ; p=0.600$ \\
Posterior deltoid & $F_{(2,12)}=0.689 ; p=0.521$ \\
Pectoralis major & $F_{(2,12)}=0.072 ; p=0.931$ \\
\hline
\end{tabular}

Several computational (Jacobs et al., 1991; Jordan and Jacobs, 1994; Wolpert and Kawato, 1998) and experimental (Ghahramani and Wolpert, 1997; Flanagan et al., 1999; Imamizu et al., 2003) studies have suggested that the brain may accomplish complex tasks by a strategy of divide and conquer. In this framework, multiple goals are simultaneously attained through the composition of control systems acting in parallel, each control system taking care of one specific feature of the composite task. Our findings are consistent with this principle. Therefore, a task involving a mixture of motion and force requirements, such as writing on a fixed or moving blackboard, may be handled by the concurrent operation of two control systems: one controlling the motion of the hand, and the other regulating the contact force. Each control system ultimately generates a force field that either drives the limb along a desired path (motion control) or delivers the desired amount of force against the environment (force control). The concurrent operation of the two control system leads to the summation of the respective fields and a production of hybrid behaviors, in which motions and contact forces are produced simultaneously. Findings from nonhuman primate studies suggest that, to maintain a continuously updated estimate of the arm state of motion (position and velocity), PPC integrates visual sensory input with previous and ongoing motor commands (Buneo and Andersen, 2006). A more recent study found that, in nonhuman primates, PPC does not encode kinetic information during reach tasks and isometric force generation tasks (HamelPâquet et al., 2006). However, during the same tasks, primary motor cortex activity is correlated with the direction and time course of force production. Our results are in agreement with these previous studies. We found that TMS of PPC selectively disrupts the force field produced by the control system during a motion tracking task but does not affect the force field produced during a force control task. Most importantly, we found that, during a task requiring both the control of hand motion and interaction force, stimulation of PPC produces an effect that is consistent with the selective disruption of the neural control of motion without interference with the control of interaction force.

Recent neuropsychological evidence has suggested separate neural encoding of forces produced during postural maintenance and arm movement (Kurtzer et al., 2005). It is tempting to interpret the results from our study in a similar context: the force control task being a postural control task equivalent to that of Kurtzer et al. (2005), and TMS of PPC causing a differential disruption of motion and not posture. However, it is important to realize that the control of posture is not equivalent to the control of static contact force. Distinctly different neural processes are recruited during static contact force control and postural stabilization (Maluf et al., 2007). The work of Kurtzer et al. provides convincing evidence that the brain separately controls postural maintenance and arm movements, while our work builds on these findings by showing that the nervous system partitions the control of isometric forces and arm movements.

In conclusion, the results we presented are not compatible with theories suggesting a common mechanism for the neural control of contact forces and arm movements. Were the nervous system implementing such a strategy, then, contrary to our findings, the disruption of hand motions would result in the concurrent disruption of contact forces. In contrast, we found new evidence supporting the notion that separate cortical circuits contribute to the control of motion and force, both separately and in tasks that require concurrent control.

\section{References}

Andersen RA, Buneo CA (2002) Intentional maps in posterior parietal cortex. Annu Rev Neurosci 25:189-220.

Bizzi E, Accornero N, Chapple W, Hogan N (1984) Posture control and trajectory formation during arm movement. J Neurosci 4:2738-2744.

Bizzi E, Hogan N, Mussa-Ivaldi F, Giszter S (1992) Does the nervous system use equilibrium-point control to guide single and multiple joint movements? Behav Brain Sci 15:603-613.

Buneo CA, Andersen RA (2006) The posterior parietal cortex: sensorimotor interface for the planning and online control of visually guided movements. Neuropsychologia 44:2594-2606.

Chouinard PA, Van Der Werf YD, Leonard G, Paus T (2003) Modulation neural networks with transcranial magnetic stimulation applied over the dorsal premotor and primary motor cortices. J Neurophysiol 90:1071-1083.

Della-Maggiore V, Malfait N, Ostry DJ, Paus T (2004) Stimulation of the posterior parietal cortex interferes with arm trajectory adjustments during the learning of new dynamics. J Neurosci 24:9971-9976.

Desmurget M, Epstein CM, Turner RS, Prablanc C, Alexander GE, Grafton ST (1999) Role of posterior parietal cortex in updating reaching movements to a visual target. Nat Neurosci 2:563-567.

Feldman A (1966a) Functional tuning of the nervous system during control of movement or maintenance of a steady posture. II. Controllable parameters of the muscles. Biophysics 11:565-578.

Feldman A (1966b) Functional tuning of the nervous system during control of movement or maintenance of a steady posture. III. Mechanographic analysis of the execution by man of the simplest motor task. Biophysics 11:766-775

Flanagan JR, Nakano E, Imamizu H, Osu R, Yoshioka T, Kawato M (1999) Composition and decomposition of internal models in motor learning under altered kinematic and dynamic environments. J Neurosci 19:RC34(1-5).

Flash T, Hogan N (1985) The coordination of arm movements: an experimentally confirmed model. J Neurosci 5:1688-1703.

Ghahramani Z, Wolpert DM (1997) Modular decomposition in visuomotor learning. Nature 386:392-395.

Giszter SF, Mussa-Ivaldi FA, Bizzi E (1993) Convergent force field organized in the frog spinal cord. J Neurosci 13:467-491.

Hamel-Pâquet C, Sergio LE, Kalaska JF (2006) Parietal area 5 activity does not reflect the differential time-course of motor output kinetics during arm-reaching and isometric-force task. J Neurophysiol 95:3353-3370.

Hogan N (1985) Impedance control: an approach to manipulation. Parts I, II, III. ASME J Dyn Syst Measurement Contr 107:1-24.

Imamizu H, Kuroda T, Miyauchi S, Yoshioka T, Kawato M (2003) Modular organization of internal models of tools in the human cerebellum. Proc Natl Acad Sci USA 100:5461-5466.

Jacobs R, Jordan M, Nowlan S, Hinton G (1991) Adaptive mixture of local experts. Neural Comput 3:79-87.

Jordan M, Jacobs R (1994) Hierarchical mixtures of experts and the EM algorithm. Neural Comput 6:181-214.

Kurtzer I, Herter TM, Scott SH (2005) Random change in cortical load representation suggests distinct control of posture and movement. Nat Neurosci 8:498-504.

Latash M (1998) Neurophysiological basis of movement. Champaign, IL: Human Kinetics.

MacDonald PA, Paus T (2003) The role of parietal cortex in awareness of self-generated movements: a transcranial magnetic stimulation study. Cereb Cortex 13:962-967.

Maluf KS, Barry BK, Riley ZA, Enoka RM (2007) Reflex responsiveness of a human hand muscle when controlling isometric force and joint position. Clin Neurophysiol 118:2063-2071. 
Mason M (1986) Mechanics and planning of manipulator pushing operations. Int J Robotics Res 5:53-71.

McIntyre J, Gurfinkel EV, Lipshits MI, Droulez J, Gurfinkel VS (1995) Measurements of human force control during a constrained arm motion using a force-actuated joystick. J Neurophysiol 73:1201-1222.

McIntyre J, Berthoz A, Lacquaniti F (1998) Reference frames and internal models. Brain Res Rev 28:143-154.

Raibert M, Craig J (1981) Hybrid position/force control of manipulators. ASME J Dyn Syst Measurement Contr 102:126-133.

Rossini PM, Barker AT, Berardelli A, Caramia MD, Caruso G, Cracco RQ, Dimitrijević MR, Hallett M, Katayama Y, Lücking CH (1994) Noninvasive electrical and magnetic stimulation of the brain, spinal cord and roots: basic principle and procedures for routine clinical application. Electroencephalogr Clin Neurophysiol 91:79-92.
Rushworth MF, Paus T, Sipila PK (2001) Attention system and the organization of the human parietal cortex. J Neurosci 21:5262-5271.

Semmler JG, Nordstrom MA (1998) Hemispheric differences in motor cortex excitability during a simple index finger abduction task in humans. J Neurophysiol 79:1246-1254.

Spong M, Hutchinson S, Vidyasagar M (1989) Robot modeling and control. New York: Wiley-Interscience Publishers.

Talairach J, Tournoux P (1998) Co-planar sterotaxic atlas of the human brain. Stuttgart, Germany: Thieme Medical Publishers.

Tunik E, Frey SH, Grafton ST (2005) Virtual lesions of the anterior intraparietal area disrupt goal-dependent on-line adjustments of grasp. Nat Neurosci 8:505-511.

Wolpert DM, Kawato M (1998) Multiple paired forward and inverse models for motor control. Neural Netw 11:1317-1329. 